\title{
UNSUPERVISED PEDESTRIAN SAMPLE EXTRACTION FOR MODEL TRAINING
}

\author{
Hao $\mathrm{Yu}^{1,2}$, Daryl Maples ${ }^{2}$, Ying Liu ${ }^{1}$ and Zhijie $\mathrm{Xu}^{2}$ \\ ${ }^{1} X i$ 'an University of Posts and Telecommunications, Xi'an, China \\ ${ }^{2}$ University of Huddersfield, Huddersfield, $U K$
}

\begin{abstract}
Many researches on pedestrian detection use benchmarking datasets such as INRIA for model training. However, models trained with standard video database do not usually obtain satisfying performance in real-life conditions. Hence, supervised training through manually labelled instances is often required to help achieving better detection result. In this research, an innovative unsupervised training approach is proposed. By analyzing the histogram of adjacent pixels modelled from the video sequences, separated pedestrians can be extracted without manual intervention. Experiments have shown consistent performance that is superior over the state-of-the-art methods.
\end{abstract}

\section{KEYWORDS}

Pedestrian Detection, Crowd Analysis, Unsupervised Learning

\section{BACKGROUND}

For approaches using classifiers for detection, the positive and negative samples are often manually labelled for model training. Most benchmarking datasets contain the annotated positive and negative samples. Thus, the acquirement of sampling information is usually ignored in the task of pedestrian detection. For example, INRIA set is used to train the detection model in approaches such as HOG (Dalal \& Triggs, 2005), FPDW (Dollar et al. 2010) and PLS (Schwartz et al. 2009). TUD-MP is used by MUTLIFTR-CSS and MUTLIFTR-MOTION (Walk et al. 2010). The PASCAL set is used by LATENT-SVM (Felzenszwalb et al. 2008). However, cameras in real-life applications are installed at varied height and angles with different perspective and effective distances. Most of the time, a model trained with samples from standard datasets do not perform as well in real-life if without additional parameter adjustment processes, which could be tedious and problem-prone. Therefore, manual labelling is often required when a new pedestrian detection system is installed. Researches have been conducted to simplify the labelling process. In the research of Davide et al. (2013), a so-called Weak-supervised annotation approach is devised to automate the extraction of positive and negative samples of pedestrians. In the work, simulated virtual video footages are firstly exploited to generate positive samples for the initial support vectors (SVM-based) training. With the trained classifier, various samples are extracted from the real-life footage and sorted with the confidence score. Next, the true positive samples of pedestrian will be manually labelled alongside the true negative samples. These weakly-supervised samples are again sent to the classifier for the second-round of training. With this process, it claims to have obtained a fine miss-rate/FPPI curve. However, the weakly-supervised labelling process is still time consuming, and requires professional personnel to operate in order to achieve the optimal detection result.

In order to address this issue, a novel unsupervised pedestrian sampling approach is proposed in this research. The devised method is capable of automatically extracting the positive and negative samples from the detected targets without the need for manual intervention. Details of the work is organized as follows: Section 2 introduced the technical design of the proposed method, including the positive and negative sampling techniques. Section 3 investigated datasets adopted for modelling and validation. The efficiency of the new approach is also analyzed in comparison with the result acquired from annotated model-based methods. Section 4 concludes the work with anticipated future improvement. 


\section{UNSUPERVISED SAMPLING OF PEDESTRIAN TARGETS}

The training of machine learning model for pedestrian detection requires two types of sampling data - positive and negative samples. The positive sample is an image patch of pedestrian with the optimal position, which could be used to extract the most representative patterns of the pedestrian. The negative samples usually are the opposite. Benchmarking dataset holds the advantage of the large number of manually labeled sample data. Take INRIA as an example, thousands of positive samples of pedestrians have been annotated.

In order to obtain as many positive samples in the unsupervised sampling process, the strategy adopted in this research is to analyze a consecutive video sequence with proper length, and to probe pedestrians who are 1) not occluded with others, 2) separable from the crowd. A histogram based positive sampling method is developed based on the principle that with sufficient video data, positive samples of large quantity can be extracted. Pilot tests demonstrated promising recall rates for extracting positive samples. In general, the number of negative samples is more important than the number of positive samples. The proposed approach consists of two parts, which are focused on the extraction of positive pedestrian samples and negative pedestrian samples separately.

\subsection{Positive Target Sampling}

The procedure of positive sampling contains three main phases. In the first phase, the foreground is subtracted from the original image. Then a histogram is modelled with the foreground pixel clusters. An average estimated cluster size to represent a pedestrian is obtained from the histogram. In the final phase, positive samples with proper foreground cluster size could be extracted from the training video sequence. The devised procedure is illustrated in the Figure 1 below.

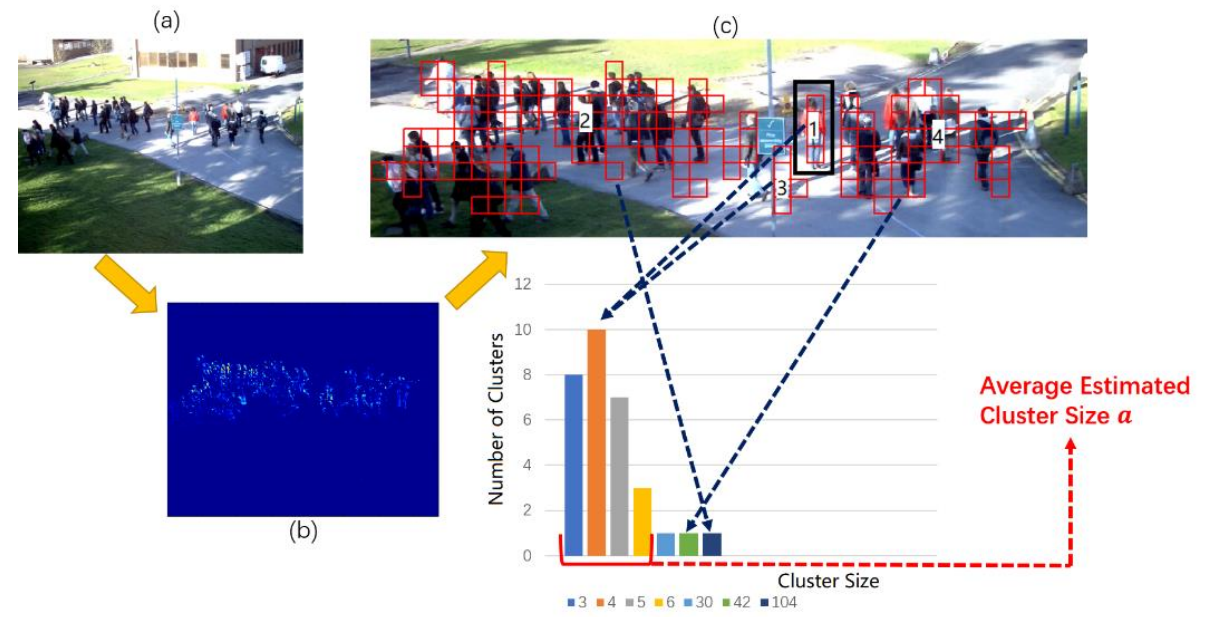

Figure 1. The procedure for estimating the average cluster size of pedestrian bounding box. (a) Original image

(b) Subtracted foreground image. (c) Clustered foreground image patches

The subtracted foreground could be obtained with either the Mixture of Gaussian segmentation (KaewTraKulPong and Bowden, 2002) or through standard threshold filtering. Next, a grid of blocks is mapped to the foreground image, any block not completely covering the foreground will be discarded. As shown in Figure 2(c) below, the red block marks the clustered foreground. A pedestrian separated from the crowd usually occupy several coherent blocks. In the training process, an image patch consists of these coherent blocks can be considered as a positive sample of a pedestrian. As shown in Figure 2(c), the pedestrian in red jacket that is marked by a black box occupies four adjacent blocks. When the position of a camera is known, one can assume that the number of coherent foreground boxes for a single pedestrian will be limited in a certain range. As aforementioned procedures that two steps will be needed to obtain various positive samples in a video sequence through, estimating of the block range, and searching for clusters within this range. 


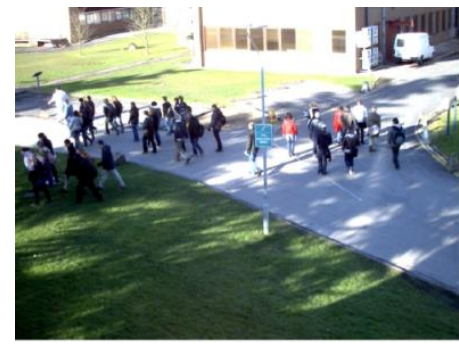

(a)

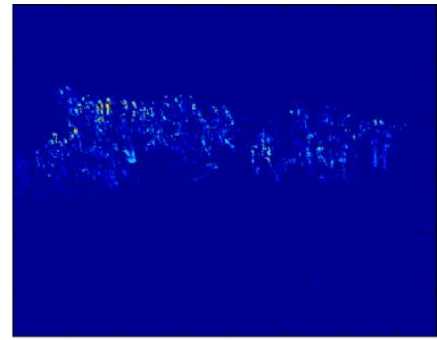

(b)

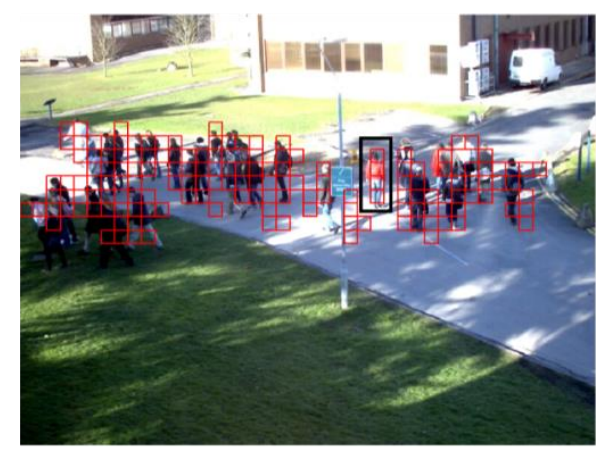

(c)

Figure 2. (a)Original image. (b) The Subtracted Foreground. (c) The marked pedestrian separated from the crowd occupies four coherent foreground blocks

\subsubsection{Estimating of the Block Range}

Based on the assumption that the cluster size for each pedestrian separated from the crowd will locate in certain range, the accumulative quantity of clusters of size within this range during certain period of time should be significantly higher than the quantity outside this range. Therefore, a histogram of cluster size and its accumulative quantity can be modelled for analysis. In order to model the histogram, adjacent blocks within a frame are labeled with numbers. As illustrated below, Figure 3(a) contains clusters with 4, 4, 104, and 42 adjacent blocks. By iteratively apply this process for several frames, a histogram can be modelled with the total number of groups of same number of blocks. The modeled histogram using 10 consecutive frames as illustrated in following Figure 3(b). It can be observed that the detected clusters with block numbers ranged from 4 to 15 are heavily grouped together and have significant higher values than clusters of different sizes. Therefore, the size of these clusters can be used to represent a separated pedestrian. Next, the average size of the cluster is calculated as the estimated patch size of a separable pedestrian target.

\subsubsection{Searching for Clusters}

Before modelling the histogram, clusters with less than 2 blocks are removed, since they are most likely generated by the background noise. Next, a 2-dimensional histogram $h=\left\{v_{1}, v_{2}, \ldots, v_{i}\right\}$ is modelled, in which $v_{i}=\left[n_{i}, m_{i}\right], n$ indicates the cluster size, and $m$ indicates the quantity of clusters with size $n$. The modelled histogram will be sparser as long as the value of $n$ become larger. The reason is when various pedestrians are occluded, the size of its derived cluster has a high probability of variation from a norm in a large scale. On the contrary, separable pedestrians in the same camera view will occupy areas within certain range. Thus, the value $m$ for separable pedestrian will be significantly larger. Next, by applying a typical classifier such as k-nearest-neighbor $(\mathrm{KNN})$ on $h$, value $n$ of this histogram can be readily classified into two clusters. It is expected that the initial $n$ with larger $m$ will be determined as the first cluster $C_{1}$ and the rest $n$ as the second cluster $C_{2}$. Once $\{v\} \in C_{1}$ is determined, the average cluster size can be estimated. The average cluster size $a$ for a single pedestrian is expressed by the following equation (1). 


$$
a=\frac{\sum_{v_{i} \in C_{1}}\left(m_{i} \times n_{i}\right)}{\sum_{v_{i} \in C_{1}} m_{i}}
$$

After the average cluster size $a$ of a separable pedestrian target is determined, any cluster of similar size will be considered as a potential pedestrian and the corresponding image patch selected as a candidate of positive sample. When the qualified clusters are located, the image patch is extracted by a bounding box with the $0.4 \mathrm{width} / \mathrm{h}$ eight ratio. The size is determined by $a$, and the center of this bounding box is set as the center of the located cluster.

The samples extracted from 27 consecutive frames from the PETS09 dataset are illustrated in Figure 4(a). During the test, 9 separable pedestrians are successfully located. Figure 4(b) shows the 2 false positives, which are caused by occlusion from the signpost. In the following process, the annotated samples will be sent to the SVM model for training.

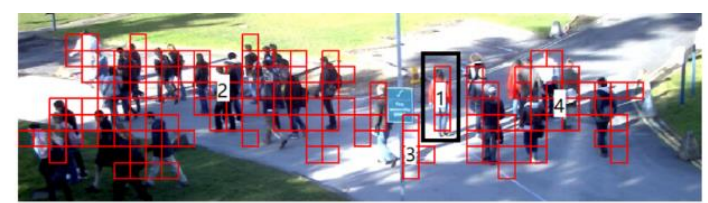

(a)

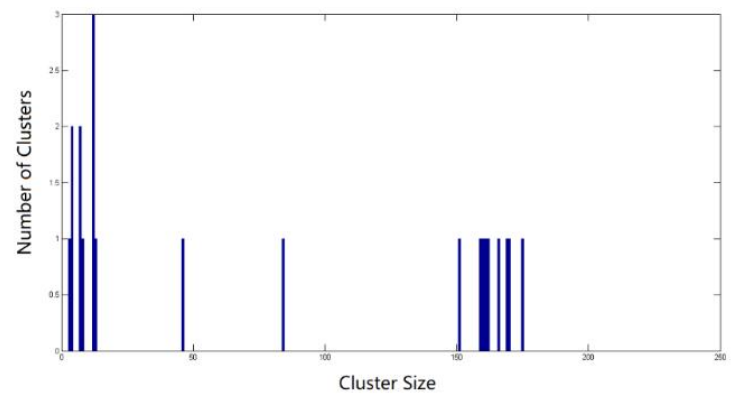

(b)

Figure 3. (a) Clustered coherent blocks. (b) Histogram modelled with clustered blocks from several consecutive frames

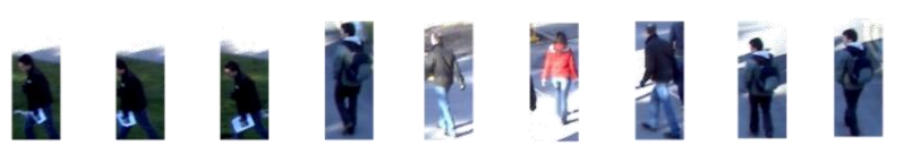

(a)

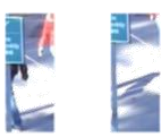

(b)

Figure 4. Extracted positive samples using the proposed approach. (a) True positive samples. (b) False positive samples

\subsection{Negative Target Sampling}

In order to improve the classification performance, the selection of negative samples is also crucial in the training phase. Most of the time, negative samples must significantly outnumber positive samples. By using a sliding window on the image, any obtained patch with certain patterns will be labelled as negative samples by the proposed approach. These patterns will be derived from 3 different circumstances, 1) a patch contains 
pure static background; 2) a patch contains part of pedestrians and part of background; 3) a patch contains multiple overlapped pedestrians.

In the first circumstance, the patch with pure background will be considered as a negative sample. Approaches on background segmentation are widely studied such as Mixture of Gaussian (MoG) (KaewTraKulPong and Bowden, 2002) (ZoranZivkovic and Ferdinandvan, 2006) (Andrew et al. 2012) and the gray-scale threshold segmentation (Milan et al. 2007). In the proposed approach, the MoG model is used for the background segmentation. In the second circumstance, the image patch contains a portion of foreground information. In this case, a threshold $T$ has been applied whose values will be estimated empirically among the range 0 to 1 . If the foreground occupies a ratio of the entire patch that is less than $T$, it will be considered as a negative sample. A practical range of $T$ is set between and 0.2 to 0.4 based on experiments. In the last circumstance, the patch contains multiple but occluded pedestrians. In this case, the patch of this type will not be qualified as an appropriate positive sample since at most of the frames, individual pedestrian do not occupy a premier lactation on the patch. However, this scenario will not be appropriate if all classified as negative samples due to it mixed nature. Thus, this type of patches will be ignored in the training phase. The following Figure 5 illustrates the negative sample selection procedures encompass different circumstances.

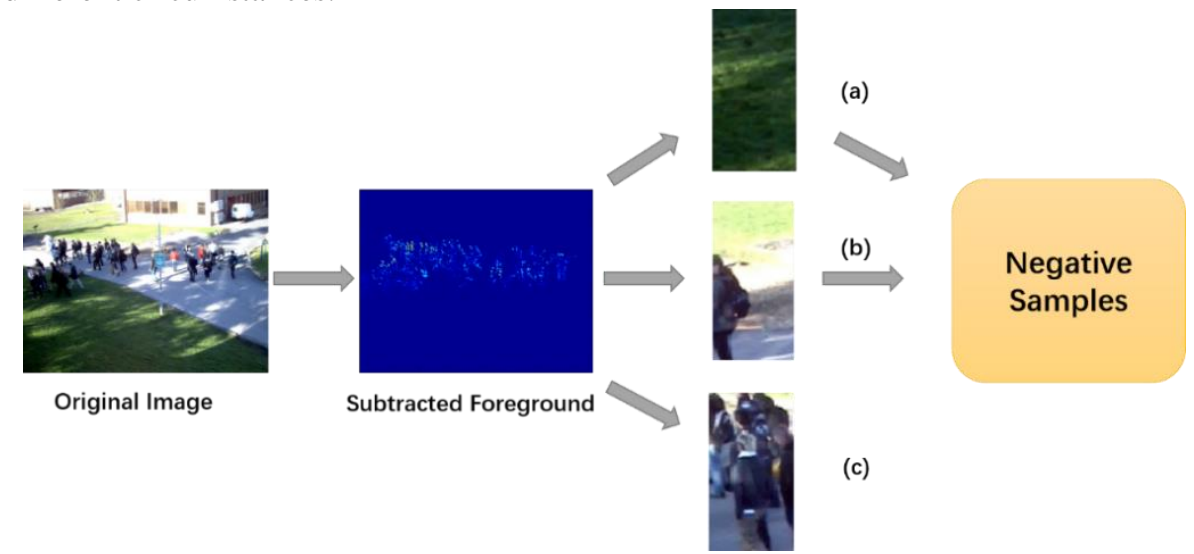

Figure 5. The procedure of selecting negative samples. (a) Pure background is categorized as solid negative. (b) Patch contains partial foreground with lower ratio than threshold will be categorized as negative. (c) Patches contain multiple overlapped objects are ignored

Once completed, a set of positive and negative samples will be automatically annotated from a video sequence and feed to classifiers for training.

\section{EXPERIMENT AND EVALUATION}

In this section, experiments are carried out to evaluate the performance of the proposed approach. In the first experiment, the efficiency of unsupervised positive sampling is assessed by benchmarking tests on four video clips from PETS2009. In the second experiment, a comparison has been made to prove the detection model trained by the unsupervised approach can outperform the model trained by the standard INRIA video dataset.

\subsection{Benchmarking and Datasets}

In the following experiments, benchmarking datasets are utilized to evaluate the performance of the developed unsupervised pedestrian detection approach. One of the most used pedestrian detection datasets is INRIA dataset (Dalal and Triggs, 2005). The INRIA set contains thousands of positive and negative data for training and testing. All pedestrians in the testing set are annotated with the co-ordinates. However, the INRIA set is not appropriate for the testing in this case due to, 1) the proposed approach is devised to handle data from the same camera that the INRIA was from different camera sources; and 2), the approach is devised to handle the pedestrian in mid-to-high density with frequent occlusive scenes that the INRIA does not satisfy. 
Another widely used dataset for pedestrian detection is PETS2009. PETS set contains crowd videos collected with static cameras from various perspectives, which is most suitable for testing the developed approach. There are three sub-categories in PETS2009 namely, person counting, tracking, and event recognition. In the person counting subset, video footages contain walking and running individuals within a mid-to-high crowd setting that are ideal for the assessment of the developed techniques and are used for both training and testing in this research.

\subsection{Positive Sample Extraction Efficiency}

In the first experiment, performance of the unsupervised sampling approach is evaluated. All 4 subsets of PETS_S1_L2 at time 14-06 are used to mine positive samples. Each subset contains 200 frames of same pedestrians from different view perspective. The sampling results vary with cameras as illustrated in Figure 6. Take camera 1 as an example, 117 samples are extracted, which consists of 55 samples of separated pedestrians and 62 samples of occluded pedestrians. 47 percent of total extracted samples are optimal/desired for training. According to the devised rule of positive sampling, most samples with clustered pedestrians are discarded. However, significant portion of samples are composed with occluded pedestrians. These samples are exploited for training process along with the desired ones, since most of them successfully captured at least a pedestrian despite of the occlusion. Another noticeable issue is the abnormality of the sample number and the low rate of desired samples. The main reason is the camera distance is too far for the accurate optical flow calculation. The inaccurate optical flow would eventually cause the false estimation of $a$, and sampling failure. This result reveals a weakness of the proposed approach that in order to achieve the best performance, the distance between camera and pedestrian need to be controlled.

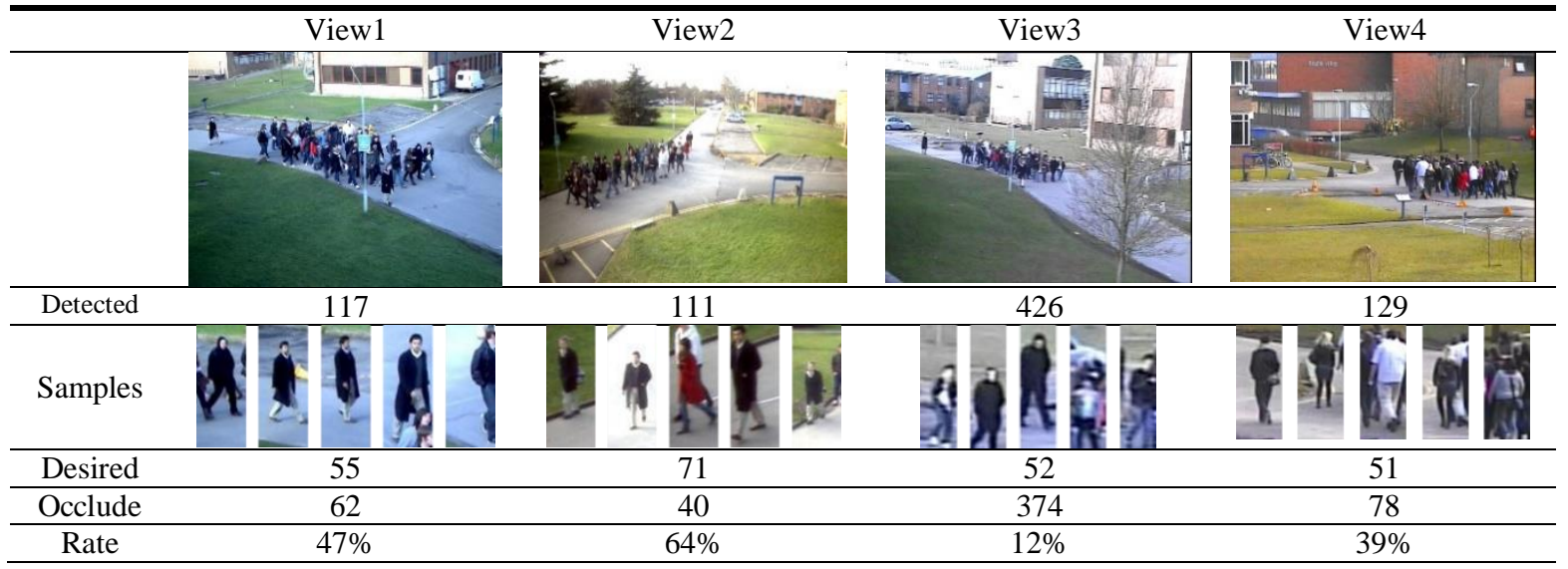

Figure 6. Sampling results of the same crowd from different cameras

\subsection{Evaluation against the Manually Labelled Dataset}

In this experiment, the performance of unsupervised sample extraction is evaluated by comparing to the detection result using the same detection procedure with manually labelled training data. Total 540 positive and 300 negative samples in a subset of INRIA are exploited to train the classifier. Figure 7 illustrates the annotation results from different perspectives of the same image frame. The results show that training using unsupervised approach is capable of achieving better performance than the standard manually based ones such as the HoG-LBP method (Xiaoyu, Tony, and Shuicheng, 2009). 


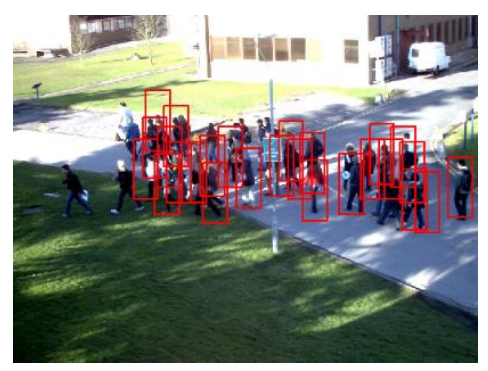

(a)

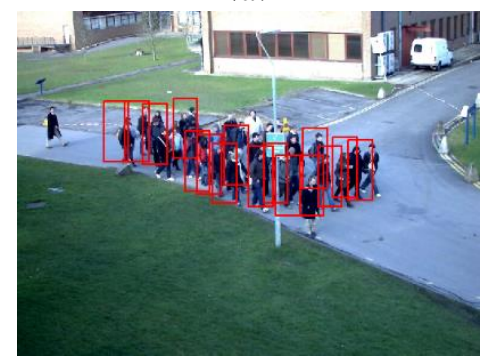

(c)

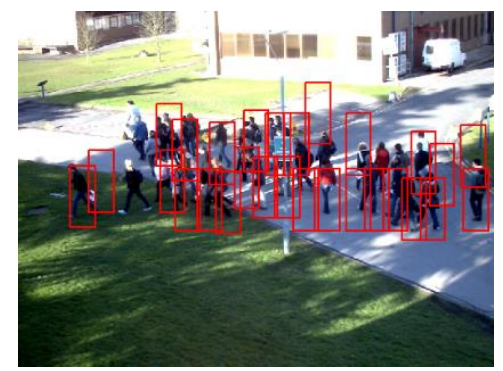

(b)

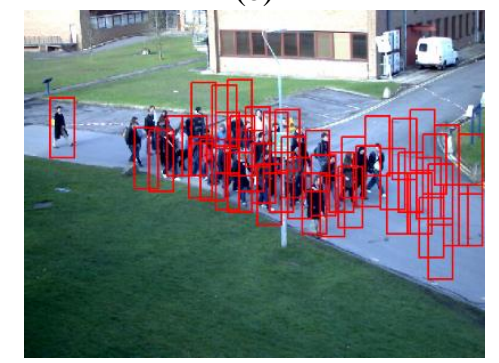

(d)

Figure 7. (a) (c) Trained with unsupervised extracted samples. (b) (d) trained with INRIA

In the next experiment, the performance of the devised approach is evaluated based on different combinations of patterns following the same unsupervised training paradigm. In the training phase, data from subset S1-L2-Time_14-06 of each camera is used for positive and negative sampling. This subset contains 200 consecutive frames, all of which will be fed to the unsupervised sampling model. The size of an image patch is set to 18 by 18 pixels. The calculated average cluster size is 10 for total 200 frames. In the detection phase, subset S1-L2-Time_14-31 is used for the detection. This subset contains 130 frames which has the identical view perspective but different illumination settings to the training data. According to the estimated pedestrian size, the bounding box is empirically set as 100 by 40 pixels. The linear SVM is trained and applied for the detection. The step of sliding window is set to 5 pixels.

As the detection result illustrated in Figure 8, the performance of pedestrian detection using unsupervised training data is slightly lower than the supervised data in all three approaches. Despite the approach using HoG-LBP pattern with Motion Rescoring and Soft-NMS techniques exhibits the best miss-rate/FPPI curve, the same approach with unsupervised training data still has high performance.

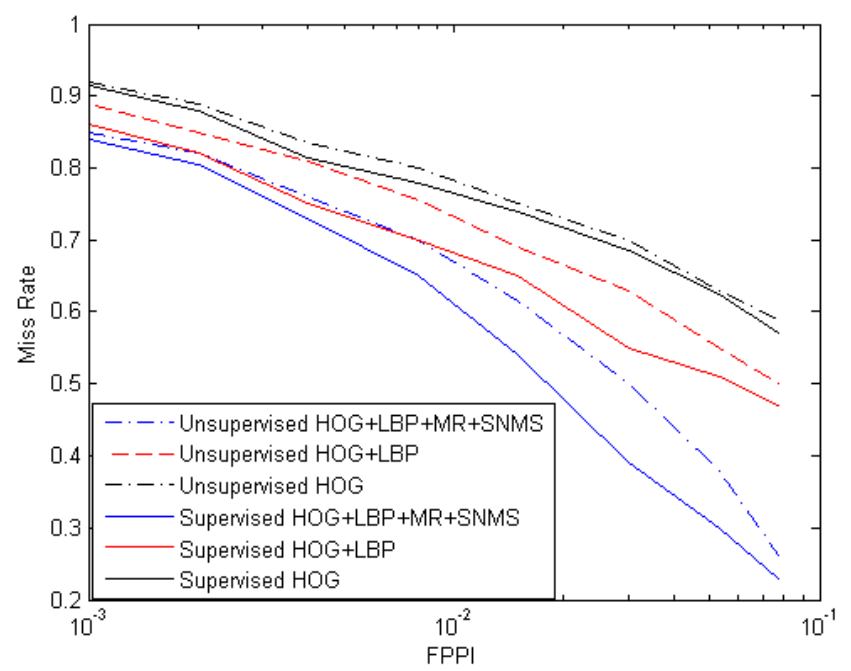

Figure 8. Comparison of detection results using the unsupervised and supervised training data 


\section{CONCLUSIONS AND FUTURE WORK}

In order to avoid the tedious and erroneous operations for training pedestrian detection systems using manually labelled dataset, this research has developed an unsupervised training approach to automatically extracting separable pedestrian targets. The experiments show the positive samples of pedestrians can be efficiently extracted by this cost-effective and efficient approach. However, due to the nature of the cluster size-based detection mechanism, the proposed solution only applies on the static camera and constant scene conditions, which is a major limit to be addressed in the further research. To improve the robustness of the proposed model, future work will see the investigation of homographic-driven frame transformation to cope with video data from different camera sources and parameter settings.

\section{ACKNOWLEDGEMENT}

This research is funded by Key Research and Development Program of Shaanxi (2019GY-054), the Sichuan Science and Technology Program (2019YFH0187) and Shaanxi Smart City Technology Project of Xianyang (No. 2017k01-25-5).

\section{REFERENCES}

Andrew, B. G. et al. (2012). Visual tracking of human visitors under variable-lighting conditions for a responsive audio art installation. In 2012 American Control Conference (ACC). Montreal, QC, Canada.

Dalal, N. and Triggs, B. (2005). Histograms of Oriented Gradients for Human Detection. In Proc. IEEE Conf. Computer Vision and Pattern Recognition, San Diego, CA, USA.

David, V. et al. (2013). Weakly Supervised Automatic Annotation of Pedestrian Bounding Boxes. In the IEEE Conference on Computer Vision and Pattern Recognition (CVPR) Workshops. Oregon, Portland.

Dollar, P. et al. (2010). The Fastest Pedestrian Detector in the West. In Proc. British Machine Vision Conf. Wales, UK.

Felzenszwalb, P. et al. (2008). A Discriminatively Trained, Multiscale, Deformable Part Model. In Proc. IEEE Conf. Computer Vision and Pattern Recognition, Anchorage, Alaska, USA.

KaewTraKulPong, P. and Bowden, R. (2002). An Improved Adaptive Background Mixture Model for Real-time Tracking with Shadow Detection. In Video-Based Surveillance Systems. Boston, MA.

Milan, S. et al. (2007). Image Processing, Analysis, and Machine Vision. Brooks/Cole, USA.

PETS 2009 Benchmark Data. Retrieved from http://www.cvg.reading.ac.uk/PETS2009/a.html

Schwartz, W. et al. (2009). Human Detection Using Partial Least Squares Analysis. In Proc. IEEE Int'l Conf. Computer Vision. Kyoto, Japan.

Walk, S. et al. (2010). New Features and Insights for Pedestrian Detection. In Proc. IEEE Conf. Computer Vision and Pattern Recognition, San Francisco, CA, USA.

Xiaoyu, W., Tony, X. H. and Shuicheng, Y. (2009). An HOG-LBP Human Detector with Partial Occlusion Handling. In the IEEE 12th International Conference on Computer Vision. Kyoto, Japan.

ZoranZivkovic, and Ferdinandvan, H. (2006). Efficient adaptive density estimation per image pixel for the task of background subtraction. Pattern Recognition Letters. 27(7), 773-780. 